\title{
Ecuación de la curva elástica
}

\author{
Yira Muñoz Sánchez ${ }^{[a]}$, Martín Ortiz Domínguez ${ }^{[a]}$, Arturo Cruz Avilés $^{[a]}$, F. Medel González ${ }^{[b]}$, A. J. Morales Robles ${ }^{[b]}$, L. E. \\ Martínez-Martínez ${ }^{[a]}$ \\ [a] Profesor Investigador de la Escuela Superior de Ciudad Sahagún en la Lic. En Ing. Mecánica-Universidad Autónoma del \\ Estado de Hidalgo \\ ${ }^{[b]}$ Estudiante de Ingeniería Mecánica en la Escuela Superior de Ciudad Sahagún de la Universidad Autónoma del Estado \\ de Hidalgo, Hidalgo, México
}

\section{Resumen}

En este trabajo de investigación se analizarán los esfuerzos y las deformaciones en elementos prismáticos sujetos a flexión. La flexión es un concepto muy importante, ya que se utiliza en el diseño de muchos componentes estructurales y de máquinas, tales como vigas y trabes. Un ejemplo de flexión pura es, por ejemplo, lo que le ocurre a una barra de una pesa gimnástica como las que sostienen los levantadores de pesas encima de su cabeza. La barra tiene pesos iguales a distancias iguales de las manos del levantador de pesas. Debido a la simetría del diagrama, las reacciones en las manos deben ser iguales y opuestas a los pesos. A pesar de lo interesantes que pueden ser las aplicaciones directas de la flexión pura, el estudio no estaría justificado si no fuera por el hecho de que los resultados obtenidos serán utilizados en el análisis de otros tipos de carga, como las cargas axiales excéntricas y las cargas transversales.

Palabras clave: Esfuerzo, Deformación unitaria, Ley de Hooke, Flexión, Constante del resorte, Modulo de elasticidad

\section{Abstract}

In this research work will be analyzed the stresses and deformations in prismatic elements subject to flexion. Flexing is a very important concept as it is used in the design of many structural components and machines, such as beams and girders. An example of pure bending is, for example, what happens to a barbell of a 
gymnastic weight such as those held by weight lifters above its head. The bar has equal weights at equal distances from the hands of the weight lifter. Because of the symmetry of the diagram, the reactions in the hands must be equal and opposite to the weights. In spite of how interesting the direct applications of pure bending can be, the study would not be justified were it not for the fact that the results obtained will be used in the analysis of other types of load, such as eccentric axial loads and cross loads.

Key words: Stress, Unitary deformation, Hooke's Law, Flexion, Spring constant, Young's modulus

\section{Introducción}

Para el estudio de Flexión pura, vamos a analizar los esfuerzos y las deformaciones en elementos prismáticos sujetos a flexión. La flexión es muy utilizado en el diseño de muchos componentes estructurales y de máquinas, tales como vigas y trabes. El análisis de elementos prismáticos sometidos a pares iguales y opuestos $M$ y $M^{\prime}$ que actúan en el mismo plano longitudinal, se dice que están sujetos a flexión pura. Los esfuerzos y deformaciones por la flexión pura en un elemento homogéneo que posea un plano de simetría y que esté elaborado de un material que siga la ley de Hooke. Un análisis preliminar de esfuerzos debidos a flexión se utilizarán métodos de estática para deducir tres ecuaciones fundamentales que deben satisfacerse por los esfuerzos normales en cualquier sección transversal dada por el elemento. Las secciones transversales permanecerán planas en un elemento sometido a flexión pura [1-6].

\section{Flexión pura}

Las deformaciones de un elemento prismático que posee un plano de simetría y está sometido en sus extremos a pares iguales y opuestos $M$ y $M^{\prime}$ que actúan en el plano de simetría. El elemento se flexionará bajo la acción de los pares, pero permanecerá simétrico con respecto a dicho plano (Figura 1). 


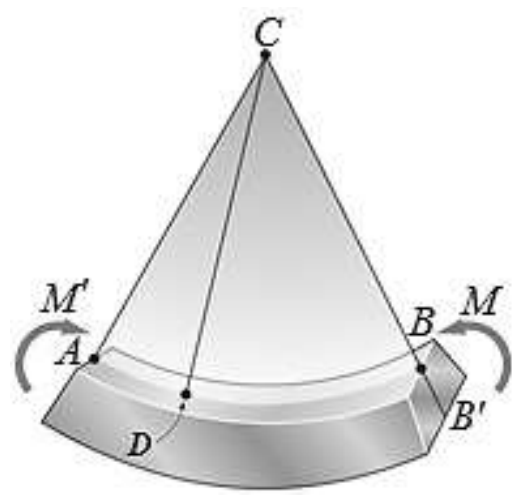

Figura 1. Sección longitudinal [1].

El momento flector $M$ es el mismo en cualquier sección, el elemento se flexionara de manera uniforme. Por lo tanto, la línea de intersección $A B$ entre la cara superior del elemento y el plano de los pares tendrá una curvatura constante. Es decir, la línea $A B$, que era originalmente recta, se transformará en un círculo de centro $\mathrm{C}$.

Se deduce que debe existir una superficie paralela a las caras superior del elemento, donde $\varepsilon_{x}$ y $\sigma_{x}$ se anulan. Esta superficie es la superficie neutra. La superficie neutra interseca el plano de simetría según un arco de circulo $D E$ (Figura 2a) e interseca una sección transversal a lo largo de una línea recta llamada eje neutro de la sección (Figura 2 b) [1-3].

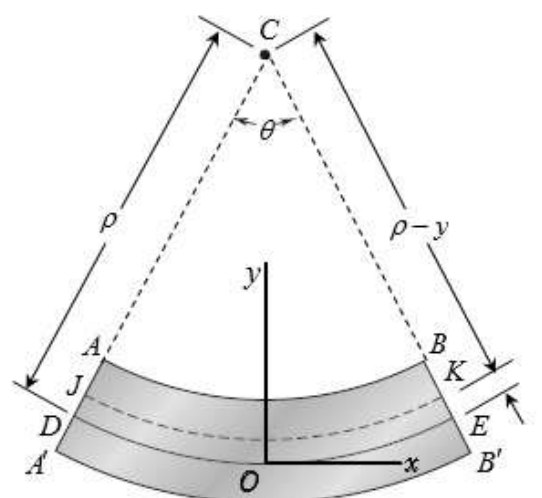

a)

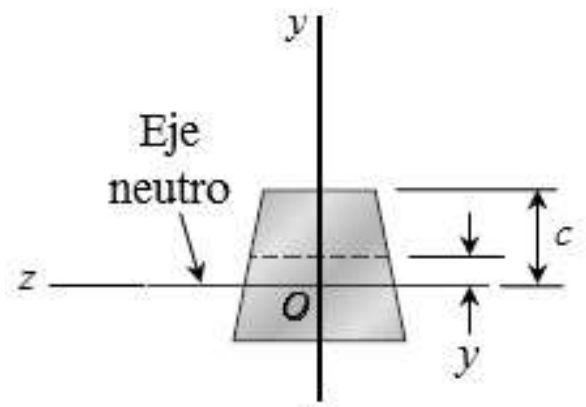

b)

Figura 2. a) Sección longitudinal, vertical (plano de simetría) y b) Sección transversal [1].

Se elegirá el origen de coordenadas en la superficie neutra, en lugar de la cara inferior, de modo que la distancia de cualquier punto a la superficie neutra se medirá por la coordenada $y$. Citando $\rho$ el radio del 
círculo $D E$ (Figura 2a), $\theta$ el ángulo central que corresponde a $D E$, y observando que la longitud de $D E$ es igual a la longitud $L$ del elemento no deformado, se tiene la Ec. (1).

$D E=L$

Considerando ahora el arco $J K$ ubicado a una distancia $y$ sobre la superficie neutra, se observa que su longitud $L^{\prime}$ es

$J K=L^{\prime}$

Asimismo se tiene que

$\tan \theta=\frac{L}{\rho}$

Reescribiendo la ec. (3) la podemos expresar de la siguiente manera

$\theta=\frac{L}{\rho}$

Despejando a $L$ tenemos:

$L=\theta \rho$

Haciendo lo mismo para obtener $L^{\prime}$

$\tan \theta=\frac{L^{\prime}}{\rho-y}$

$\theta=\frac{L^{\prime}}{\rho-y}$

$L^{\prime}=\theta(\rho-y)$ 
Como a longitud original del arco $J K$ era igual a $L$, la deformación $\delta$ es igual a:

$\delta=L^{\prime}-L$

Sustituyendo las Ecs. (5) y (8) en la Ec. (9), se obtiene que:

$\delta=\theta(\rho-y)-\theta \rho$

Multiplicando la Ec. 10, se llega a:

$\delta=\theta \rho-\theta y-\theta \rho$

Reescribiendo la Ec. 11, se tiene:

$\delta=-\theta y$

Recordando la Ecuación de la deformación unitaria longitudinal, se tiene que:

$\varepsilon_{x}=\frac{\delta}{L}$

Sustituyendo las Ecs. (5) y (8) en la Ec. (9), se obtiene que:

$\varepsilon_{x}=\frac{-\theta y}{\theta \rho}=-\frac{y}{\rho}$

La deformación unitaria $\varepsilon_{x}$, alcanza su máximo valor absoluto cuando $y$ es máxima, si $c$ es la distancia máxima a la superficie neutra (que corresponde a la superficie superior o inferior del elemento), y $\varepsilon_{x}^{\max }$ es el máximo valor absoluto de la deformación unitaria.

$\varepsilon_{x}^{\max }=\frac{c}{\rho}$ 
Reescribiendo la Ec. (14), se tiene:

$\frac{1}{\rho}=-\frac{\varepsilon_{x}}{y}$

Asimismo la Ec. (15), se puede reescribir

$$
\frac{1}{\rho}=\frac{\varepsilon_{x}^{\max }}{c}
$$

Igualando las Ecs. (16) y (17), se tiene lo siguiente:

$-\frac{\varepsilon_{x}}{y}=\frac{\varepsilon_{x}^{\max }}{c}$

Resolviendo la Ec. (18) para la deformación unitaria longitudinal $\varepsilon_{x}$, se puede escribir como:

$\varepsilon_{x}=-\frac{y \varepsilon_{x}^{\max }}{c}$

Por otro lado, los esfuerzos y deformaciones en el rango elástico se pueden apreciar en la Figura 3:

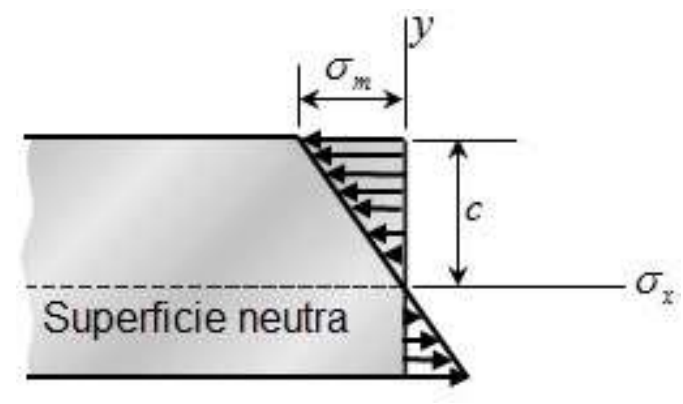

Figura 3. Esfuerzo y deformaciones en el rango elástico [1]. 
El esfuerzo en la dirección $x$, se expresa como:

$\sigma_{x}=E \varepsilon_{x}$

Multiplicando por $E$ a la Ecuación (19), se obtiene:

$E \varepsilon_{x}=-E \frac{y \varepsilon_{x}^{\max }}{c}$

Considerando la Ec. (20), en la Ec. (21), se llega a:

$\sigma_{x}=-\frac{y}{c} \sigma_{x}^{\max }$

Recordando que el esfuerzo normal es igual a:

$\sigma_{x}=\frac{F_{x}}{A}$

Asimismo:

$\sigma_{x}=\frac{d F_{x}}{d A}$

Igualando las Ecs. (22) y (24)

$\frac{d F_{x}}{d A}=-\frac{y}{c} \sigma_{x}^{\max }$

$d F_{x}=-\frac{y}{c} \sigma_{x}^{\max } d A$ 
Integrando la ecuación y multiplicado por $-y$ en ambos lados dela ecuación se tiene que:

$$
-\int_{0}^{A} y \sigma_{x} d A=\frac{\sigma_{x}^{\max }}{c} \int_{0}^{A} y^{2} d A=M
$$

Recordando que:

$$
\int_{0}^{A} y^{2} d A=I
$$

Sustituyendo la Ec. (28) en la Ec. (27), se llega a:

$$
\frac{\sigma_{x}^{\text {máx }} I}{c}=M
$$

Y por lo tanto el esfuerzo máximo en $x$ es igual a:

$$
\sigma_{x}^{m a ́ x}=\frac{M c}{I}
$$

Asimismo:

$$
\sigma_{x}^{\max }=\frac{M y}{I}
$$

Para la obtención de la ecuación de la curva elástica tenemos que:

$$
\varepsilon_{x}^{\text {máx }}=\frac{c}{\rho}
$$


$\frac{1}{\rho}=\frac{\varepsilon_{x}^{\max }}{c}$

Reescribiendo la ley de Hooke, se tiene:

$\sigma_{x}^{\text {máx }}=E \varepsilon_{x}^{\text {máx }}$

$\varepsilon_{x}^{\text {máx }}=\frac{\sigma_{x}^{\text {máx }}}{E}$

Sustituyendo la Ec. (30) en la Ec. (35), del esfuerzo máximo anteriormente encontrada, tenemos que:

$\varepsilon_{x}^{\max }=\frac{M c}{E I}$

Ahora sustituyendo la deformación unitaria máxima tenemos que:

$\frac{c}{\rho}=\frac{M c}{E I}$

Para la obtención de la ecuación diferencial de la curva elástica tenemos la siguiente figura: 


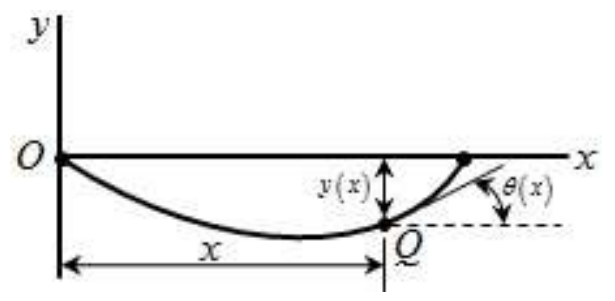

Figura 4. Elemento diferencial de la viga para la determinación de la ecuación diferencial de la curva elástica [1].

Por otro lado, considerando la Figura 4, se tiene:

$\tan \theta=\frac{y}{x}$

$\tan \theta=\frac{d y}{d x}$

Tomando en cuenta que la $\tan \theta=\theta$

$\theta=\frac{d y}{d x}$

Ahora derivamos el ángulo respecto a $x$ :

$$
\begin{aligned}
& \frac{d \theta}{d x}=\frac{d}{d x}\left(\frac{d y}{d x}\right) \\
& \frac{d \theta}{d x}=\frac{d^{2} y}{d x^{2}}
\end{aligned}
$$

Tomando en cuenta de la Figura 4 que: $d s=\rho d \theta$ 
$\frac{1}{\rho}=\frac{d \theta}{d s}$

Considerando que $d s \approx d x$, entonces:

$\frac{1}{\rho}=\frac{d \theta}{d x}=\frac{d^{2} y}{d x^{2}}$

Recordando que $\frac{1}{\rho}=\frac{M}{E I}$, tenemos que:

$\frac{d^{2} y}{d x^{2}}=\frac{M}{E I}$

Obtenemos la ecuación diferencial de la curva elástica. Las condiciones adicionales de frontera requeridas pueden obtenerse observando que aunque la fuerza cortante y el momento flector pueden ser discontinuos en varios puntos de una viga, la deflexión y la pendiente de la viga no pueden ser discontinuas en ningún punto.

\section{Conclusión}

La flexión es muy usada para determinar las propiedades de los materiales como son flexibilidad y ductilidad. El ensayo de flexión se basa en la aplicación de una fuerza puntual y simplemente apoyada, para determinar la resistencia del material hacia una carga estática o aplicada lentamente. Normalmente se usa para materiales tenaces, y los más importante nos ayuda a determinar la magnitud de la fuerza a la cual el material fallara.

\section{Agradecimientos}


El trabajo descrito en el presente artículo fue financiado por la beca otorgada por PRODEP. Asimismo, los autores le agradecen al Dr. Jorge Zuno Silva, quien es Director de la Escuela Superior de Ciudad SahagúnUAEH, México, por todas las facilidades para concluir este trabajo de investigación.

\section{Bibliografía}

${ }^{[1]}$ Beer, F.P.,Johnston, E.R,(2010), “Mecánica de Materiales”, MacGraw-Hill. 5ta Edición

${ }^{[2]}$ Pytel, A., Singer, F. L., (1987), “Resistencia de Materiales", Oxford, 4ta Edición

${ }^{[3]}$ Hibbeler, R.C,(1995), “Mecánica de Materiales”, Prentice Hall. 3ra Edición

${ }^{[4]}$ Norton, R. L. (1999). Diseño de Máquinas. Estado de México, México: Prentice Hall Hispanoamérica, S. A.

${ }^{[5]}$ Shigley, J. E., and Mischke, C. R. (1989). Mechanical Engineering Design. New York, US: MacGraw-Hill. 